\title{
Description of New Piezoresistance Tensor Equation for Cubic Single Crystal and Its Application to Multiaxial Stress
}

\author{
Taeko Ando ${ }^{*}$ and Toshiyuki Toriyama \\ Department of Mechanical Engineering, College of Science and Engineering Ritsumeikan University, \\ 1-1-1 Noji-higashi, Kusatsu, Shiga 525-8577, Japan
}

(Received April 6, 2018; accepted August 6, 2018)

Keywords: piezoresistance, cubic single crystal, multiaxial stress, tensor equation

In this paper, we derived a new piezoresistance tensor equation for a cubic single crystal. This equation can be expressed in terms of the three independent principal components of piezoresistance tensors, the isotropic and deviatoric stress tensors, and the fourth-rank coordinate transformation tensor. The piezoresistance tensor equation can be decomposed into a relation between only the hydrostatic part of the stress tensor and the trace of the resistivity change tensor and into a relation between only the deviatoric parts. The hydrostatic part of the piezoresistance tensor equation is invariant with respect to a coordinate transformation. On the other hand, the deviatoric part of the tensor equation is traceless. The proposed piezoresistance tensor decomposition gives a new physical insight into the classical theory of Pfann and Thurston [J. Appl. Phys. 32 (2008) 1961]. It was shown that Pfann and Thurston's theory can be rewritten as a special case of our tensor decomposition. Furthermore, to demonstrate the consistency between the proposed tensor equation and the experimental evidence, some basic experiments on a single-crystal silicon piezoresistive rosette stress gauge subjected to multiaxial stress were carried out.

\section{Introduction}

The piezoresistance in various types of cubic single crystals is widely used as a forcesensing element in micro-electromechanical systems (MEMS). ${ }^{(1-5)}$ The large piezoresistance in single-crystal silicon was discovered by Smith in 1954, ${ }^{(6)}$ who described some of the basic properties of the piezoresistance from the viewpoint of the fourth-rank tensor field. ${ }^{(7)}$ In the same era, Pfann and Thurston proposed a theory of single-crystal silicon piezoresistive stress transducers considering the anisotropy of cubic crystals. ${ }^{(8)}$ Tufte and Stelzer performed important experiments on a heavily doped single-crystal silicon piezoresistive stress gauge produced with an integrated circuit (IC)-compatible fabrication process..$^{(9,10)}$ Their results have been widely used as a design standard for single-crystal silicon piezoresistive stress transducers on IC chips. Since then, the number of published papers concerning cubic-single-crystal piezoresistive stress transducers has increased yearly. ${ }^{(1-5)}$ The successful launch of cubic-

*Corresponding author: e-mail: tando@fc.ritsumei.ac.jp

https://doi.org/10.18494/SAM.2018.1959 
single-crystal piezoresistive stress transducers into the MEMS market is due to the huge amount of accumulated data and the IC-compatible microfabrication process. An excellent review by Barlian et al. described the research trend of applying cubic-single-crystal semiconductor piezoresistance to microsystem technology over half a century. ${ }^{(3)}$

The classical theory of Pfann and Thurston has been widely supported by practical engineers designing cubic-single-crystal piezoresistive stress transducers. ${ }^{(3)}$ Pfann and Thurston adopted the Voigt notation to describe the components of the cubic piezoresistance tensor. They derived 21 components of the piezoresistance tensor in terms of three independent principal components and the direction cosine between the principal crystallographic orientation and an arbitrary crystallographic orientation. The combination of the 21 components of the piezoresistance tensor and the six components of the stress tensor leads to six piezoresistance equations containing the state of multiaxial stress and an arbitrary crystallographic orientation. However, the obtained mathematical structure of the piezoresistance is very complex and it is difficult to find the general physical role of the state of multiaxial stress in the cubic crystal anisotropy of the piezoresistance. A plausible reason for the lack of physical significance of Pfann and Thurston's theory is the adoption of components in the analysis of piezoresistance. It is well recognized that the analysis of piezoresistance based on its components and coefficients is very useful for specific engineering designs and applications, such as specific stress states, crystallographic planes, and orientations in advance. However, the analysis of piezoresistance in terms of its components gives an unclear physical relationship between the state of multiaxial stress and the crystal anisotropy of the piezoresistance with respect to a coordinate transformation.

Therefore, in this paper, we derive a single piezoresistance tensor equation that clearly indicates the physical role of the state of multiaxial stress in the cubic crystal anisotropy of the piezoresistance. Both the fourth-rank piezoresistance tensor and the second-rank stress tensor are decomposed into isotropic (hydrostatic) and deviatoric parts. Then, the piezoresistance tensor equation is decomposed into isotropic and deviatoric parts. The piezoresistance tensor equation can also be decomposed into a relation between only the hydrostatic part of the stress tensor and the trace of the resistivity change tensor and another relation between only the deviatoric parts. The hydrostatic part of the piezoresistance tensor equation is invariant with respect to a coordinate transformation. On the other hand, the deviatoric part of the tensor equation is traceless. These are new findings in the theory of piezoresistance. The piezoresistance tensor equation gives a new physical insight into the typical single-crystal silicon piezoresistive stress transducers derived by Pfann and Thurston. ${ }^{(8)}$ It was shown that Pfann and Thurston's theory can be rewritten as a special case of our tensor decomposition. The cubic-single-crystal piezoresistance may be regarded as a revisited problem, ${ }^{(7-10)}$ but our sophisticated method gives a new physical insight into the theory of cubic crystal piezoresistance and into practical design applications, as briefly demonstrated in the appropriate sections in this paper. Furthermore, to demonstrate the consistency between the tensor equation and the experimental evidence, some basic experiments on a single-crystal silicon piezoresistive stress gauge subjected to multiaxial stress were carried out. 
The organization of our paper is as follows. In the first part of this paper, we derive a single piezoresistance tensor equation. It is shown that this equation can be expressed in terms of the three independent principal components of piezoresistance tensors, the isotropic (hydrostatic) and deviatoric stress tensors, and the fourth-rank coordinate transformation tensor. In addition, the physical structure of the cubic piezoresistance subjected to an arbitrary plane stress is clarified under a coordinate transformation. The obtained result will be useful for MEMS piezoresistive mechanical sensor design because a single-crystal silicon wafer is the standard substrate for the fabrication process and the MEMS piezoresistive mechanical sensor is subjected to a plane stress condition in most applications.

In the second part of this paper, the piezoresistance measurement of a single-crystal silicon microbeam element subjected to multiaxial stress is carried out. The microfabrication process and experimental procedure for the single-crystal silicon piezoresistive multiaxial stress rosette gauge on the microbeam element are briefly described. To demonstrate the mathematical and physical validity of the proposed concept, experimental results are compared with a theoretical prediction based on the tensor equation. It is shown that the decomposition of the piezoresistance tensor can give new physical insight into the classical theory of Pfann and Thurston (hereafter referred to as the PT theory). The basic concept of the PT theory is to form a piezoresistor that responds only to specified stress components. Regarding this point, the PT theory provides no physical basis to find a combination of appropriate crystallographic orientations that exhibits sensitivity to the specified stress components. It is demonstrated that making a piezoresistor unresponsive to specified stress components is equivalent to finding an appropriate form of the deviatoric part of the piezoresistance tensor equation that can cancel the specified stress components appearing in the hydrostatic part of it.

\section{Description of Piezoresistance Tensor Equation for Cubic-Single-Crystal Symmetry}

\subsection{Piezoresistance tensor equation for cubic-crystal symmetry}

We define two Cartesian coordinate systems corresponding to the arbitrary crystallographic frame $\mathrm{O}-x_{1} x_{2} x_{3}$ and the principal cubic-single-crystal crystallographic frame $\mathrm{O}-X_{1} X_{2} X_{3}$. The orthogonal orientations of the two Cartesian coordinate systems can be related by three Euler angles $(\psi, \theta, \phi)$. If an index occurs twice in any term of the Cartesian tensor components, summation is taken from 1 to 3 . However, the tensor summation convention does not apply to the Greek indices. Furthermore, the capital letters in the summation symbol obey the ordinary summation rule and the tensor summation convention does not apply to them. According to a previous work, ${ }^{(11)}$ a piezoresistance tensor having cubic anisotropy with respect to the cubicsingle-crystal crystallographic principal frame $\mathrm{O}-X_{1} X_{2} X_{3}$ can be written as

$$
\Pi_{i j k l}=\hat{\Pi}_{1} \delta_{i j} \delta_{k l}+2 \hat{\Pi}_{2} I_{i j k l}+\hat{\Pi}_{3} \sum_{L=1}^{3} \delta_{i L} \delta_{j L} \delta_{k L} \delta_{l L},
$$


where $\delta_{i j}$ is the Kronecker delta, $I_{i j k l}=\frac{1}{2}\left(\delta_{i k} \delta_{j l}+\delta_{i l} \delta_{j k}\right)$,

$$
\begin{aligned}
& \hat{\Pi}_{1}=\Pi_{1122}=\Pi_{2211}=\Pi_{2233}=\Pi_{3322}=\Pi_{3311}=\Pi_{1133}, \\
& \hat{\Pi}_{2}=\Pi_{2323}=\Pi_{3131}=\Pi_{1212} \\
& \left(\Pi_{2323}=\Pi_{2332}=\Pi_{3223}=\Pi_{3232} \text {, and similarly for other components, } \Pi_{3131} \text { and } \Pi_{1212}\right), \\
& \hat{\Pi}_{1}+2 \hat{\Pi}_{2}+\hat{\Pi}_{3}=\Pi_{1111}=\Pi_{2222}=\Pi_{3333} .
\end{aligned}
$$

Note that the anisotropy factors defined as $\hat{\Pi}_{1}, \hat{\Pi}_{2}$, and $\hat{\Pi}_{3}$ are measures of the anisotropy of crystals, and these are standard descriptions for the elastic compliance and stiffness tensors. The Voigt notation reduces the fourth-rank tensor components to second-rank components as $\Pi_{1111}=\Pi_{11}, \Pi_{1122}=\Pi_{12}$, and $\Pi_{2323}=\frac{\Pi_{44}}{2}$. The law of the fourth-rank tensor transformation leads to the following piezoresistance tensor with respect to the arbitrary crystallographic frame:

$$
\pi_{i j k l}=\Pi_{p q r s} \frac{\partial X_{p}}{\partial x_{i}} \frac{\partial X_{q}}{\partial x_{j}} \frac{\partial X_{r}}{\partial x_{k}} \frac{\partial X_{s}}{\partial x_{l}}
$$

where $\frac{\partial X_{p}}{\partial x_{i}}$ is the direction cosine between the reference frames O- $X_{1} X_{2} X_{3}$ and O- $x_{1} x_{2} x_{3}$, which can be expressed by a standard method using the three Euler angles $(\psi, \theta, \phi)$ (Fig. 1) as follows. ${ }^{(12)}$

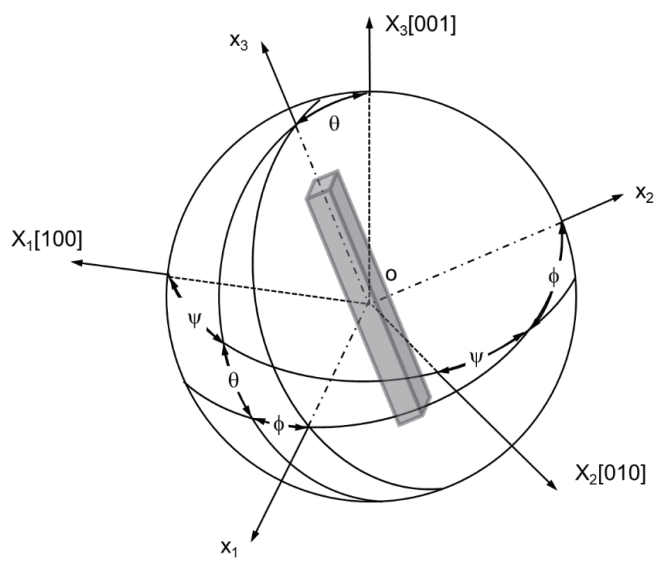

Fig. 1. (Color online) Three Euler angles $(\psi, \theta, \phi)$ used to define the direction cosine tensor component $\frac{\partial X_{p}}{\partial x_{i}}$ between the piezoresistor reference frame $\mathrm{O}-X_{1} X_{2} X_{3}$ and the crystallographic principal frame $\mathrm{O}-x_{1} x_{2} x_{3}$. 


$$
\begin{array}{cccc} 
& x_{1} & x_{2} & x_{3} \\
X_{1} & a_{11}=\cos \phi \cos \theta \cos \psi & a_{12}=\cos \phi \cos \theta \sin \psi & a_{13}=-\cos \phi \sin \theta \\
& -\sin \phi \sin \psi & +\sin \phi \cos \psi & \\
X_{2} & a_{21}=-\sin \phi \cos \theta \cos \psi & a_{22}=-\sin \phi \cos \theta \sin \psi & a_{23}=\sin \phi \sin \theta \\
X_{3} & -\cos \phi \sin \psi & +\cos \phi \cos \psi & \\
& a_{31}=\sin \theta \cos \psi & a_{32}=\sin \theta \sin \psi & a_{33}=\cos \theta
\end{array}
$$

The substitution of Eq. (1) into Eq. (2) leads to the following piezoresistance tensor with respect to the arbitrary crystallographic frame $\mathrm{O}-x_{1} x_{2} x_{3}$ :

$$
\pi_{i j k l}=\hat{\Pi}_{1} \delta_{i j} \delta_{k l}+2 \hat{\Pi}_{2} I_{i j k l}+\hat{\Pi}_{3} L_{i j k l}
$$

where $L_{i j k l} \equiv \sum_{L=1}^{3} a_{L i} a_{L j} a_{L k} a_{L l}$ is a fourth-rank tensor that consists of the second-rank tensors $a_{L i} \equiv \frac{\partial X_{L}}{\partial x_{i}}$ given in Eq. (3). The fourth-rank tensor $L_{i j k l}$ can be decomposed into isotropic and deviatoric parts as (see Appendix A)

$$
L_{i j k l}=\left(L_{i j k l}\right)_{\text {isotropic }}+\left(L_{i j k l}\right)_{\text {deviatoric }} \equiv\left(L_{i j k l}\right)_{I}+\left(L_{i j k l}\right)^{*}
$$

where

$$
\begin{aligned}
& \left(L_{i j k l}\right)_{i s o t r o p i c} \equiv\left(L_{i j k l}\right)_{I}=L_{1} \delta_{i j} \delta_{k l}+2 L_{2} I_{i j k l}=\frac{1}{5}\left(\delta_{i j} \delta_{k l}+2 I_{i j k l}\right), \\
& L_{1}=\frac{1}{15}\left(2 L_{p p q q}-L_{p q p q}\right)=\frac{1}{5}, \text { and } L_{2}=\frac{1}{10}\left(L_{p q p q}-\frac{1}{3} L_{p p q q}\right)=\frac{1}{5} .
\end{aligned}
$$

The piezoresistance tensor $\pi_{i j k l}$ can be decomposed into isotropic and deviatoric parts in a similar way to Eq. (5) (see Appendix A):

$$
\pi_{i j k l}=\left(\pi_{i j k l}\right)_{i s o t r o p i c}+\left(\pi_{i j k l}\right)_{\text {deviatoric }} \equiv\left(\pi_{i j k l}\right)_{I}+\left(\pi_{i j k l}\right)^{*}
$$

where

$$
\begin{aligned}
& \left(\pi_{i j k l}\right)_{\text {isotropic }}=\left(\pi_{i j k l}\right)_{I}=\left(\hat{\Pi}_{1}+\frac{1}{5} \hat{\Pi}_{3}\right) \delta_{i j} \delta_{k l}+2\left(\hat{\Pi}_{2}+\frac{1}{5} \hat{\Pi}_{3}\right) I_{i j k l}, \\
& \left(\pi_{i j k l}\right)_{\text {deviatoric }}=\left(\pi_{i j k l}\right)^{*}=\hat{\Pi}_{3}\left[L_{i j k l}-\frac{1}{5}\left(\delta_{i j} \delta_{k l}+2 I_{i j k l}\right)\right] .
\end{aligned}
$$


The stress tensor can be decomposed into isotropic (spherical) and deviatoric parts by referring to the classical continuum theory of plasticity as follows: ${ }^{(13)}$

$$
\sigma_{i j}=\left(\sigma_{i j}\right)_{\text {isotropic }}+\left(\sigma_{i j}\right)_{\text {deviatoric }} \equiv \sigma \delta_{i j}+\left(\sigma_{i j}\right)^{*}
$$

where $\sigma \equiv \frac{1}{3} \sigma_{p p}$ is the scalar-valued mean stress related to the first stress invariant, and $\left(\sigma_{i j}\right)^{*}$ is the deviatoric stress tensor whose trace is $\left(\sigma_{p p}\right)^{*}=0$.

The generalized Ohm's law with respect to the arbitrary crystallographic frame $\mathrm{O}-x_{1} x_{2} x_{3}$ is expressed as ${ }^{(7)}$

$$
e_{i}=\left(\rho_{0} \delta_{i k}+\Delta \rho_{i k}\right) j_{k},
$$

where $e_{i}$ is the electrical field vector, $j_{k}$ is the current density vector, $\rho_{0}$ is the scalar invariant of the zero-stress resistivity, and $\Delta \rho_{i k}$ is the resistivity change tensor due to the applied stress. When stress is applied to a cubic anisotropic medium with respect to the arbitrary crystallographic frame $\mathrm{O}-x_{1} x_{2} x_{3}$, the second-rank tensor fields of stress and resistivity change are connected at every point by the linear relation ${ }^{(3,6)}$

$$
\frac{\Delta \rho_{i j}}{\rho_{0}}=\pi_{i j k l} \sigma_{k l}
$$

where $\frac{\Delta \rho_{i j}}{\rho_{0}}$ is the relative resistivity change tensor due to the applied stress.

The substitution of Eqs. (6) and (7) into Eq. (9) leads to the decomposition of the piezoresistance tensor equation into the hydrostatic and deviatoric parts of stress tensors,

$$
\begin{aligned}
\frac{\Delta \rho_{i j}}{\rho_{0}} & =\frac{1}{3} \frac{\Delta \rho_{m m}}{\rho_{0}} \delta_{i j}+\left(\frac{\Delta \rho_{i j}}{\rho_{0}}\right)^{*} \\
& =\left(3 \hat{\Pi}_{1}+2 \hat{\Pi}_{2}+\hat{\Pi}_{3}\right) \sigma \delta_{i j}+2 \hat{\Pi}_{2}\left(\sigma_{i j}\right)^{*}+\hat{\Pi}_{3} L_{i j k l}\left(\sigma_{k l}\right)^{*} \\
& =\left(\Pi_{11}+2 \Pi_{12}\right) \sigma \delta_{i j}+\Pi_{44}\left(\sigma_{i j}\right)^{*}+\left(\Pi_{11}-\Pi_{12}-\Pi_{44}\right) L_{i j k l}\left(\sigma_{k l}\right)^{*},
\end{aligned}
$$

where $\frac{1}{3} \frac{\Delta \rho_{m m}}{\rho_{0}} \delta_{i j} \equiv\left(3 \hat{\Pi}_{1}+2 \hat{\Pi}_{2}+\hat{\Pi}_{3}\right) \sigma \delta_{i j}$ is the hydrostatic part and $\left(\frac{\Delta \rho_{i j}}{\rho_{0}}\right)^{*} \equiv 2 \hat{\Pi}_{2}\left(\sigma_{i j}\right)^{*}+\hat{\Pi}_{3} L_{i j k l}\left(\sigma_{k l}\right)^{*}$ is the deviatoric part. 
An alternative derivation of Eq. (10) is useful to clarify its physical meaning. Therefore, we give a brief discussion below. We note again that if an index occurs once in any term of the Cartesian tensor components, the index is free and is taken from 1 to 3 , and if an index occurs twice in any term of the Cartesian tensor components, the index is dummy and summation is taken from 1 to 3 . A scalar multiple of $\pi_{m m n n}$ and $\delta_{i j}$ forms the isotropic secondrank piezoresistance tensor $\pi_{i j m m}=\frac{1}{3} \pi_{m m n n} \delta_{i j}$. The fourth-rank piezoresistance tensor $\pi_{i j k l}$ that becomes the zero second-rank tensor when its suffixes are contracted, i.e., $\pi_{m m k l}=\pi_{k l m m}$ $=0$, is defined as the traceless fourth-rank piezoresistance tensor (deviatoric tensor) $\left(\pi_{i j k l}\right)^{*}$. Consequently, the number of independent components is no more than 15 for the symmetric fourth-rank tensor, i.e., $\pi_{i j k l}=\pi_{k l i j}$. The decomposition of the fourth-rank piezoresistance tensor $\pi_{i j k l}$ is straightforward,

$$
\pi_{i j k l}=\frac{1}{9} \pi_{m m n n} \delta_{i j} \delta_{k l}+\left(\pi_{i j k l}\right)^{*}
$$

where $\left(\pi_{m m k l}\right)^{*}=\left(\pi_{k l m m}\right)^{*}=0$.

By introducing the traceless parts of the stress and resistivity change tensors, i.e., $\left(\sigma_{i j}\right)^{*}=\sigma_{i j}-\frac{1}{3} \sigma_{p p} \delta_{i j}$ and $\left(\Delta \rho_{i j}\right)^{*}=\rho_{i j}-\frac{1}{3} \rho_{p p} \delta_{i j}$, Eq. (9) can be broken down as

$$
\begin{aligned}
\frac{1}{3} \frac{\Delta \rho_{p p}}{\rho_{0}} \delta_{i j} & =\frac{1}{3} \pi_{m m n n} \sigma_{p p} \delta_{i j}, \\
\left(\frac{\Delta \rho_{i j}}{\rho_{0}}\right)^{*} & =\left(\pi_{i j k l}\right)^{*}\left(\sigma_{k l}\right)^{*} .
\end{aligned}
$$

Therefore, the piezoresistance tensor equation can be decomposed into a relation between only the hydrostatic part of the stress tensor (the trace of the stress tensor) and the trace of the resistivity change tensor and into another relation between only the deviatoric parts. The tensor Eqs. (10) and (12) have clear physical meanings. The deviatoric part of Eqs. (10) and (12) becomes the zero second-rank tensor when its suffixes are contracted, i.e., $2 \hat{\Pi}_{2}\left(\sigma_{m m}\right)^{*}+\hat{\Pi}_{3} L_{m m k l}\left(\sigma_{k l}\right)^{*}=\left(2 \hat{\Pi}_{2}+\hat{\Pi}_{3}\right)\left(\sigma_{m m}\right)^{*}=0$ and $\left(\pi_{m m k l}\right)^{*}\left(\sigma_{k l}\right)^{*}=0$. On the other hand, the isotropic parts of Eqs. (10) and (12) represent the relation between only the hydrostatic part of the stress tensor and the trace of the resistivity change tensor. Therefore, the trace of Eqs. (10) and (12) leads to the following scalar invariant with respect to the coordinate transformation.

$$
\frac{\Delta \rho_{m m}}{\rho_{0}}=\left(\Pi_{11}+2 \Pi_{12}\right) \sigma_{m m}
$$


The geometrical explanation of Eq. (13) has not been well recognized. The relative change in resistance in a series-connected configuration of three orthogonal piezoresistors is proportional to the hydrostatic part of the stress tensor and insensitive to the three principal stress orientations as shown in Fig. 2.

If a multiaxial stress is applied to a cubic anisotropic medium with respect to the cubicsingle-crystal crystallographic principal frame $\mathrm{O}-X_{1} X_{2} X_{3}$, then the component of the deviatoric stress tensor with respect to the arbitrary crystallographic frame $\mathrm{O}-x_{1} x_{2} x_{3}$ is transformed as

$$
\left(\sigma_{i j}^{\prime}\right)^{*}=a_{k i} a_{l j}\left(\sigma_{k l}\right)^{*}
$$

The combination of Eqs. (10) and (14) leads to the following component of the piezoresistance tensor equation with respect to the arbitrary crystallographic frame $\mathrm{O}-x_{1} x_{2} x_{3}$ as a function of the stress components with respect to the cubic-single-crystal crystallographic principal frame $\mathrm{O}-X_{1} X_{2} X_{3}$ :

$$
\begin{aligned}
\frac{\Delta \rho_{i j}^{\prime}}{\rho_{0}} & =\left(3 \hat{\Pi}_{1}+2 \hat{\Pi}_{2}+\hat{\Pi}_{3}\right) \sigma \delta_{i j}+2 \hat{\Pi}_{2} a_{k i} a_{l j}\left(\sigma_{k l}\right)^{*}+\hat{\Pi}_{3} \sum_{L=1}^{3} a_{L i} a_{L j}\left(\sigma_{L L}\right)^{*} \\
& =\left(\Pi_{11}+2 \Pi_{12}\right) \sigma \delta_{i j}+\Pi_{44} a_{k i} a_{l j}\left(\sigma_{k l}\right)^{*}+\left(\Pi_{11}-\Pi_{12}-\Pi_{44}\right) \sum_{L=1}^{3} a_{L i} a_{L j}\left(\sigma_{L L}\right)^{*} .
\end{aligned}
$$

A piezoresistor subjected to a remote uniform uniaxial normal stress along the principal cubicsingle-crystal crystallographic frame is transformed to the state of multiaxial stress by the

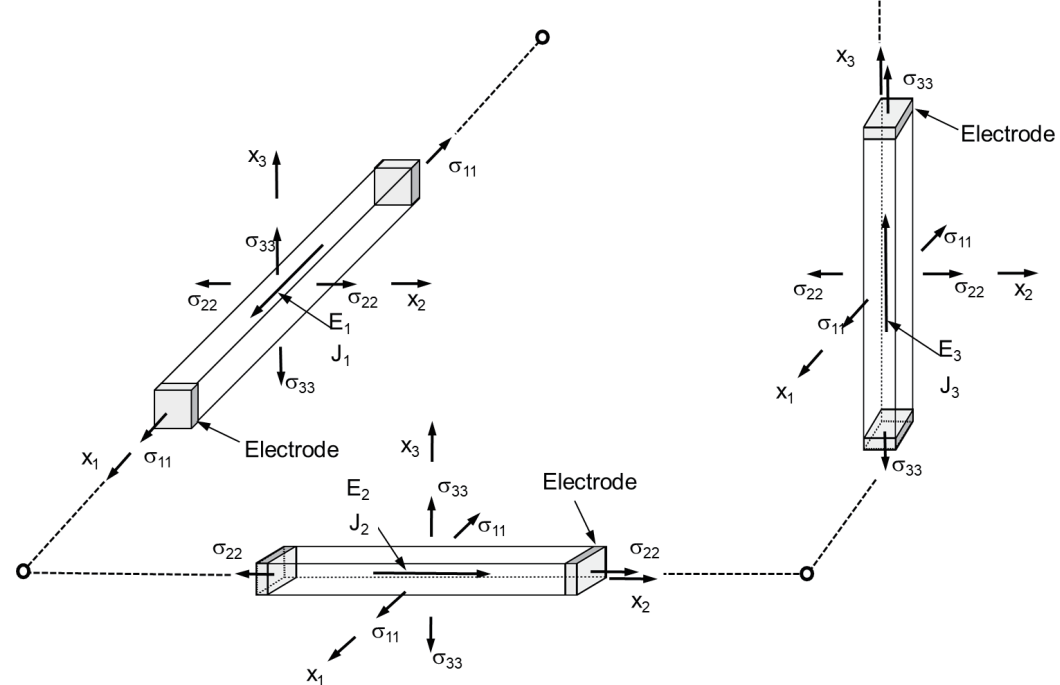

Fig. 2. Schematic of the series-connected three-orthogonal-piezoresistor (the relative change in resistance is proportional to the trace of the stress tensor and insensitive to the three principal stress orientations). 
coordinate transformation and vice versa. Therefore, such a consideration is useful for the design of MEMS piezoresistive mechanical sensors on single-crystal silicon wafers subjected to arbitrary plane stress. As a special case, if we consider the in-plane ( $X_{1} X_{2}$-plane) state of multiaxial stress with respect to the principal cubic-single-crystal crystallographic frame O- $X_{1} X_{2} X_{3}$, then the relative resistance change $\frac{\Delta R_{11}^{\prime}}{R_{0}}$ in a piezoresistor in which the current density and electrical field are both parallel to an arbitrary $x^{\prime}{ }_{1}$ direction on the crystallographic plane ( $X_{1} X_{2}$-plane) can be given by Eqs. (10) and (15), neglecting the dimensional change due to the elastic deformation [this will be briefly discussed as Eq. (17) in Sect. 2.2].

$$
\frac{\Delta R_{11}^{\prime}}{R_{0}}=\left(\frac{\Delta R_{11}^{\prime}}{R_{0}}\right)_{\text {hydrostatic }}+\left(\frac{\Delta R_{11}^{\prime}}{R_{0}}\right)_{\text {deviatoric }},
$$

where

$$
\begin{aligned}
&\left(\frac{\Delta R_{11}^{\prime}}{R_{0}}\right)_{\text {hydrostatic }} \cong \frac{1}{3} \frac{\Delta \rho_{11}}{\rho_{0}} \delta_{11}=\frac{1}{3}\left(\Pi_{11}+2 \Pi_{12}\right)\left(\sigma_{11}+\sigma_{22}\right), \\
&\left(\frac{\Delta R_{11}^{\prime}}{R_{0}}\right)_{\text {deviatoric }} \cong\left(\frac{\Delta \rho_{11}}{\rho_{0}}\right)^{*} \\
&=-\frac{1}{3}\left(\Pi_{11}-\Pi_{12}\right)\left(a_{11}^{2}+a_{21}^{2}\right)\left(\sigma_{11}+\sigma_{22}\right) \\
&+\left(\Pi_{11}-\Pi_{12}\right)\left(a_{11}^{2} \sigma_{11}+a_{21}^{2} \sigma_{22}\right)+2 \Pi_{44} a_{11} a_{21} \sigma_{12} .
\end{aligned}
$$

These results are used for the discussion of piezoresistance measurement.

\subsection{Geometrical effect of resistance change due to linear elastic deformation}

We derive a general equation for the effect of the relative resistance change due to elastic deformation. This correction was first discussed by Smith. ${ }^{(6)}$ We derive the following general tensor equation valid for an arbitrary crystallographic coordinate transformation:

$$
\frac{\Delta R_{i j}}{R_{0}}=\frac{\Delta \rho_{i j}}{\rho_{0}}+2 \varepsilon_{i j}-\varepsilon_{p p},
$$

where $\varepsilon_{i j}$ is the linear elastic strain tensor and $\varepsilon_{p p}$ is the trace of $\varepsilon_{i j}$ with reference to the dilatation of normal strain. Equation (17) is only valid for the normal strain $(i=j)$ because the shear strain $(i \neq j)$ does not change the volume of a solid and makes no contribution to the resistance change due to elastic deformation.

The linear elastic strain tensor is related to the stress tensor by Hooke's law: 


$$
\varepsilon_{i j}=s_{i j k l} \sigma_{k l},
$$

where $s_{i j k l}$ is the elastic compliance tensor.

The crystal symmetry of the cubic elastic compliance tensor is similar to that of the piezoresistance tensor. Therefore, applying Nye's expression to the Voigt notation, ${ }^{(14,15)}$ we can derive

$$
s_{i j k l}=\hat{S}_{1} \delta_{i j} \delta_{k l}+2 \hat{S}_{2} I_{i j k l}+\hat{S}_{3} L_{i j k l}
$$

where $3 \hat{S}_{1}+2 \hat{S}_{2}+\hat{S}_{3} \equiv S_{11}+2 S_{12}, 2 \hat{S}_{2} \equiv \frac{1}{2} S_{44}, 2 \hat{S}_{2}+\hat{S}_{3} \equiv S_{11}-S_{12}$.

The substitution of Eqs. (18) and (19) into Eq. (17) leads to the following relative resistance change due to the applied stress in place of the elastic strain:

$$
\begin{aligned}
2 \varepsilon_{i j}-\varepsilon_{p p}= & \left(S_{11}+2 S_{12}\right)\left(2 \delta_{i j}-3\right) \sigma+S_{44}\left(\sigma_{i j}\right)^{*} \\
& +2\left(S_{11}-S_{12}-\frac{1}{2} S_{44}\right) L_{i j k l} \sigma_{k l}^{*},
\end{aligned}
$$

where $i=j$.

Figure 3 depicts graphical representations of $\left(2 \varepsilon_{11}-\varepsilon_{p p}\right) / \sigma_{11}$ for single-crystal silicon subjected to the in-plane uniaxial tension $\sigma_{11}$ with respect to the arbitrary crystallographic orientation in the $\{100\},\{110\}$, and $\{111\}$ planes. The values of the three components of the cubic elastic compliance tensor, i.e., $S_{11}, S_{12}$, and $S_{44}$, were taken from Ref. 15 and used for the calculation of $\left(2 \varepsilon_{11}-\varepsilon_{p p}\right) / \sigma_{11}$ from Eq. (20). As can be seen from the figures, it is straightforward to derive Smith's geometrical correction as a special case of Eq. (20). The graphical variation of $\left(2 \varepsilon_{11}-\varepsilon_{p p}\right) / \sigma_{11}$ depicted in Fig. 3 is similar to that of the Young's modulus ${ }^{(15)}$ because the in-plane uniaxial tensile strain $\varepsilon_{11}$ applied to the piezoresistor is directly related to the in-plane uniaxial tensile stress $\varepsilon_{11}$. The order of $\left(2 \varepsilon_{11}-\varepsilon_{p p}\right) / \sigma_{11}$ for single-crystal silicon is $\left(2 \varepsilon_{11}-\varepsilon_{p p}\right) / \sigma_{11} \sim 1 / S_{11} \sim 10^{-12}(1 / \mathrm{Pa})$. On the other hand, the orders of the piezoresistance tensor components $\Pi_{11}, \Pi_{12}$, and $\Pi_{44}$ for single-crystal silicon are $\Pi_{11}, \Pi_{12}$, and $\Pi_{44} \sim 10^{-9}(1 / \mathrm{Pa})$, respectively. Therefore, the effect of the relative resistance change due to elastic deformation can be neglected for single-crystal silicon.

\section{Piezoresistance Measurement}

A piezoresistive rosette stress gauge consisting of seven p-type piezoresistors was prepared on an n-type (100) silicon substrate. Each piezoresistor had a length of $100 \mu \mathrm{m}$, a width of 10 $\mu \mathrm{m}$, and a depth of $2 \mu \mathrm{m}$. The shape of the piezoresistive rosette stress gauge formed a right angle. The longitudinal directions of the two end piezoresistors coincided with the $\langle 110>$ crystallographic orientation. The right angle was divided into $15^{\circ}$ steps from the end as shown 


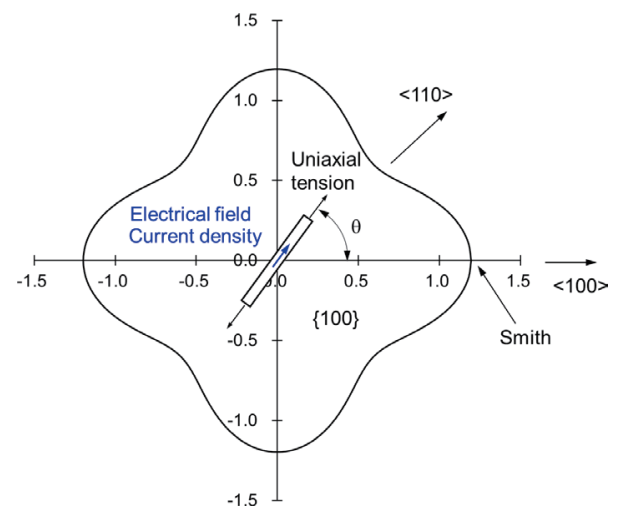

(a)

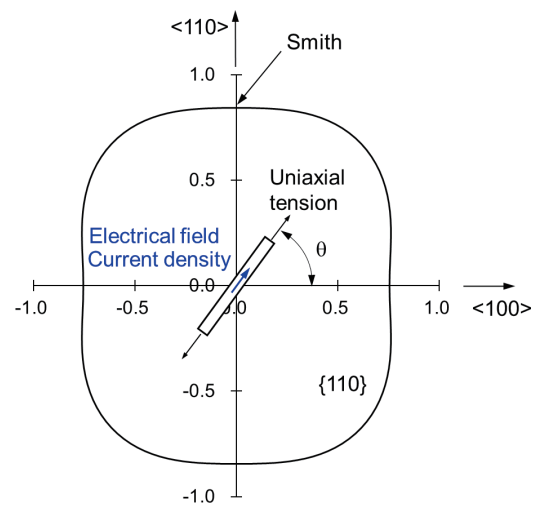

(b)

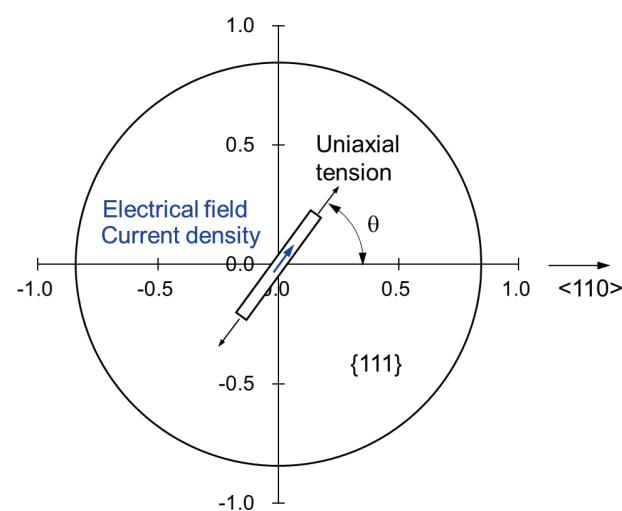

(c)

Fig. 3. (Color online) Graphical representations of the relative resistance change $\left(2 \varepsilon_{11}-\varepsilon_{p p}\right) / \sigma_{11}$ due to the inplane uniaxial tension $\sigma_{11}$ with respect to the arbitrary crystallographic orientation in $\{100\},\{110\}$, and $\{111\}$ planes of the single-crystal silicon (unit: $\times 10^{-11} \mathrm{~Pa}^{-1}$ ).

in Fig. 4. The piezoresistive rosette stress gauge was fabricated by a combination of twostep thermal diffusion and ultraviolet lithography. The sheet resistance of the p-type silicon diffusion layer was $52.6 \Omega / \square$ and the surface carrier concentration was approximately $10^{18} \mathrm{~cm}^{-3}$. A rectangular microbeam was cut from the n-type (100) silicon substrate and used to remotely apply the uniform tensile stress $\sigma_{0}$ along the $<110>$ direction to the piezoresistive rosette stress gauge via a simple four-point bending system (Fig. 5).

As previously mentioned in the discussion of Eq. (16), the relative resistance change in a piezoresistor in which the directions of the current density and electrical field are both parallel to an arbitrary $x^{\prime}{ }_{1}$ direction in the crystallographic plane $\left(X_{1} X_{2}\right.$-plane) is

$$
\begin{aligned}
\frac{\Delta R_{11}^{\prime}}{R_{0}} \cong & \frac{1}{3}\left(\Pi_{11}+2 \Pi_{12}\right)\left(\sigma_{11}+\sigma_{22}\right)-\frac{1}{3}\left(\Pi_{11}-\Pi_{12}\right)\left(a_{11}^{2}+a_{21}^{2}\right)\left(\sigma_{11}+\sigma_{22}\right) \\
& +\left(\Pi_{11}-\Pi_{12}\right)\left(a_{11}^{2} \sigma_{11}+a_{21}^{2} \sigma_{22}\right)+2 \Pi_{44} a_{11} a_{21} \sigma_{12} .
\end{aligned}
$$

Therefore, the response of the piezoresistive rosette stress gauge depends on the three plane 


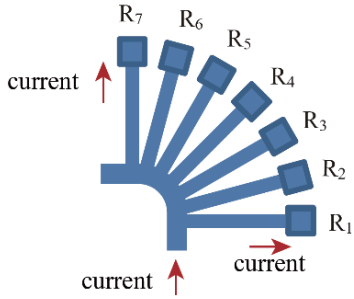

\begin{tabular}{lr}
\hline Dimension & $(\mu \mathrm{m})$ \\
\hline Length & 100 \\
Width & 10 \\
Depth of resistor & 2 \\
\hline
\end{tabular}

Fig. 4. (Color online) Schematic of the piezoresistive rosette stress gauge that consists of seven p-type piezoresistors on the n-type (100) silicon substrate.
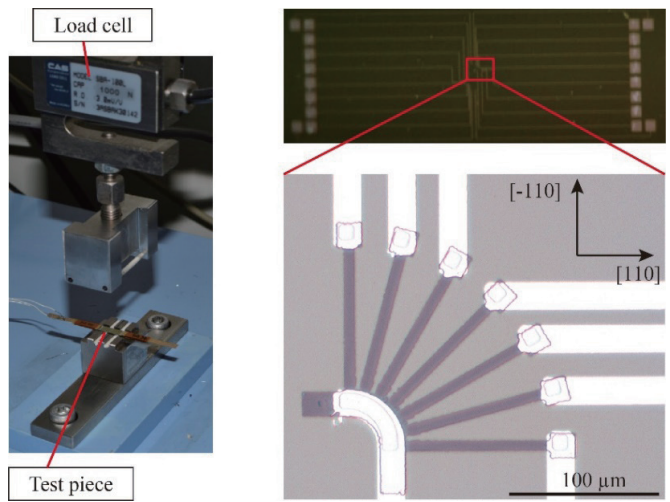

Fig. 5. (Color online) Rectangular microbeam of the n-type (100) silicon substrate used to apply the uniform remote tensile stress $\sigma_{0}$ along $<110>$ direction to the piezoresistive rosette stress gauge via a simple four-point bending.

stress components $\sigma_{11}, \sigma_{22}$, and $\sigma_{12}$ applied to the principal crystallographic axis in the $\{100\}$ plane. It is straightforward to derive Eq. (14) in Ref. 16 as a special case of Eq. (21).

Figure 6 shows the relationship between the transformation of the stress component and the crystallographic orientation subjected to uniform remote uniaxial tensile stress $\sigma_{0}$ along $<110>$ in the $\{100\}$ plane. The constitutive equation for this experiment can be rewritten from Eq. (21) with Euler angles $(\psi=0, \theta=0)$ and direction $\operatorname{cosines} a_{11}=\cos \phi$ and $a_{21}=-\sin \phi$.

$$
\frac{\Delta R_{11}^{\prime}}{R_{0}} \cong \frac{\Pi_{11}+2 \Pi_{12}}{3} \sigma_{0}-\left(\frac{\Pi_{11}-\Pi_{12}}{6}-\frac{\Pi_{44}}{2}\right) \sigma_{0} \sin 2 \phi,
$$

where $\left(\frac{\Delta R_{11}^{\prime}}{R_{0}}\right)_{\text {hydrostatic }} \cong \frac{\Pi_{11}+2 \Pi_{12}}{3} \sigma_{0}$ and $\left(\frac{\Delta R_{11}^{\prime}}{R_{0}}\right)_{\text {deviatoric }} \cong-\left(\frac{\Pi_{11}-\Pi_{12}}{6}-\frac{\Pi_{44}}{2}\right) \sigma_{0} \sin 2 \phi$, and $-\frac{\pi}{4} \leq \phi \leq \frac{\pi}{4}, \phi= \pm \frac{\pi}{4}$ coincides with $<110>$, and $\phi=0$ coincides with $<100>$ as shown in Fig. 6. If we perform the superposition and replacement of the remote stress as $\sigma_{11}=\sigma_{22}=\frac{\sigma_{0}}{2}$ and $\sigma_{12}=+\frac{\sigma_{0}}{2}$, then the alternative derivation of Eq. (22) from Eq. (21) is straightforward.

A semiconductor parameter analyzer (HP4155A) was used to measure the resistance change in the piezoresistive rosette stress gauge. Figure 7 shows a comparison of the normalized theoretical equation $\frac{\Delta R_{11}^{\prime}}{\left|\Delta R_{11}^{\prime}\right|_{\max }}$ based on Eq. (22) and the experimental results. The theoretical equation was in good agreement with the experimental values. Thus, the part of the proposed theory was experimentally verified. 


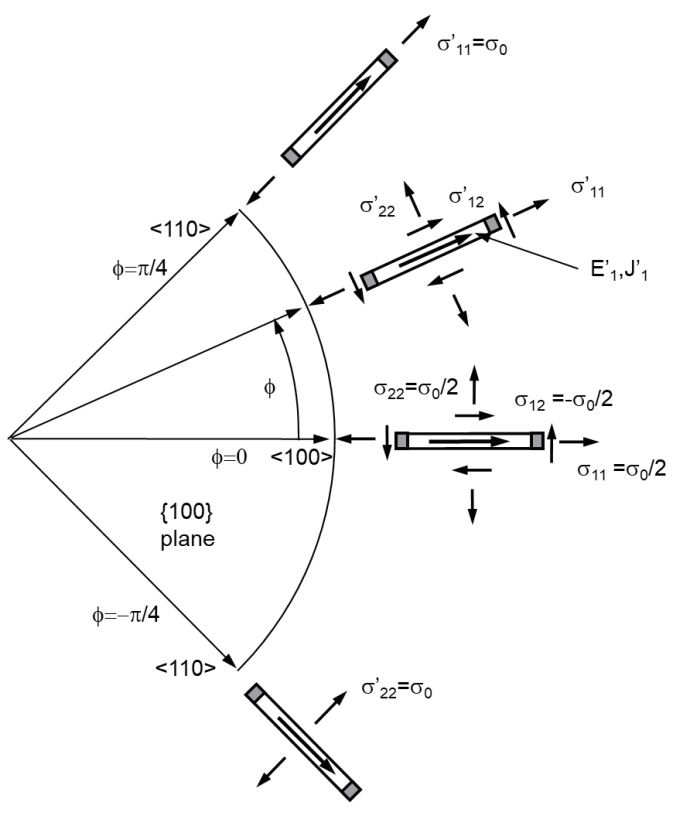

Fig. 6. Relationship between the stress component transformation and the crystallographic orientation under the uniform remote tensile stress $\sigma_{0}$ along $<110>$ direction in $\{100\}$ plane depicted in Fig. 4.

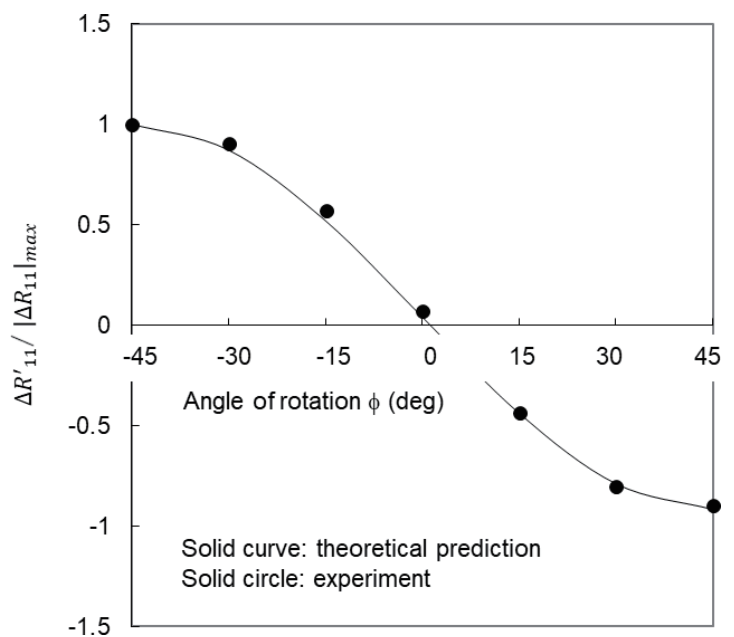

Fig. 7. Comparison of the normalized theoretical equation of $\frac{\Delta R_{11}^{\prime}}{\left|\Delta R_{11}^{\prime}\right|_{\max }}$ and the experimental results.

\section{Physical Interpretation of PT Theory Based on the New Piezoresistance Tensor Equation}

The validity of the proposed theory was verified by experimental evidence in the previous section. Here, we demonstrate that the design of a piezoresistor derived by using PT theory can be reproduced as a special case of our piezoresistance equation. We primarily discuss a piezoresistor fabricated by the planar diffusion technique on the surface of bulk single-crystal silicon and a piezoresistor fabricated on silicon-on-insulator (SOI) substrates as described in the previous section. We assume that the in-plane normal and shear stresses are faithfully transmitted to the piezoresistors as assumed by Pfann and Thurston. ${ }^{(8)}$

\subsection{Tensor equation for piezoresistor responding only to specified stress components}

The basic concept of the PT theory is to form a piezoresistor that responds only to specified stress components. Regarding this point, the PT theory provides no physical basis to find a combination of appropriate crystallographic orientations that exhibit sensitivity to the specified stress components. We start from Eq. (10) to provide a new physical basis in a manner unified with the PT theory. 


$$
\begin{aligned}
\frac{\Delta \rho_{\alpha \alpha}}{\rho_{0}}= & \frac{1}{3}\left(\Pi_{11}+2 \Pi_{12}\right) \sigma_{k k}+\Pi_{44}\left(\sigma_{\alpha \alpha}-\frac{1}{3} \sigma_{k k}\right) \\
& +\left(\Pi_{11}-\Pi_{12}-\Pi_{44}\right) L_{\alpha \alpha i j}\left(\sigma_{i j}-\frac{1}{3} \sigma_{k k} \delta_{i j}\right),
\end{aligned}
$$

Note that the tensor summation convention does not apply to the Greek indices. Equation (23) corresponds to the configuration of a piezoresistor subjected to multiaxial stress, where the electrical field and current density vectors are both parallel to the $x_{\alpha}$ direction. By setting $L_{\alpha \alpha 12}=L_{\alpha \alpha 21}=0, L_{\alpha \alpha 23}=L_{\alpha \alpha 32}=0$, and $L_{\alpha \alpha 31}=L_{\alpha \alpha 13}=0$, the crystallographic orientations for which the piezoresistor does not respond to the shear stress components can be obtained from Eq. (23). The hydrostatic part of Eq. (23), i.e., $\frac{1}{3}\left(\Pi_{11}+2 \Pi_{12}\right) \sigma_{k k}$, is scalar-invariant and responds to the first stress invariant regardless of the crystallographic orientation. Therefore, making a piezoresistor unresponsive to specified stress components is equivalent to finding an appropriate form of the deviatoric part of Eq. (23) that can cancel the specified stress components appearing in the hydrostatic part of Eq. (23). If we design a piezoresistor that does not respond to the stress component $\sigma_{\beta \beta}$, then the general procedure is to find a combination of appropriate crystallographic orientations $L_{\alpha \alpha \beta \beta}$ that cancel the stress component of $\sigma_{\beta \beta}$ in the hydrostatic part (see Appendix B), where $L_{\alpha \alpha \beta \beta}$ is given by

$$
\begin{gathered}
L_{\alpha \alpha \beta \beta}=-\frac{\Pi_{12}}{\Pi_{11}-\Pi_{12}-\Pi_{44}} \text { for } \alpha \neq \beta, \\
L_{\alpha \alpha \beta \beta}=-\frac{\Pi_{12}+\Pi_{44}}{\Pi_{11}-\Pi_{12}-\Pi_{44}} \text { for } \alpha=\beta .
\end{gathered}
$$

\subsection{Tensor equation for piezoresistor insensitive to stress orientation}

The removal of the third piezoresistor from the three-piezoresistor configuration results in a two-piezoresistor configuration. This configuration is a special case of the series-connected three-piezoresistor configuration represented by Eq. (13) (Fig. 2), which is scalar-invariant with respect to the coordinate transformation. Equation (23) leads to

$$
\frac{\Delta \rho_{33}}{\rho_{0}}=\Pi_{12}\left(\sigma_{11}+\sigma_{22}\right)+\left(\Pi_{11}-\Pi_{12}-\Pi_{44}\right)\left(L_{3311} \sigma_{11}+L_{3322} \sigma_{22}\right),
$$

which corresponds to the configuration of a piezoresistor subjected to in-plane biaxial normal stress with the electrical field and current density vectors both parallel to the plane normal direction $x_{\alpha}$. Therefore, the general equation corresponding to the two-piezoresistor configuration is 


$$
\begin{aligned}
\frac{\Delta \rho_{m m}}{\rho_{0}}-\frac{\Delta \rho_{33}}{\rho_{0}}= & \left(\Pi_{11}+\Pi_{12}\right)\left(\sigma_{11}+\sigma_{22}\right) \\
& -\left(\Pi_{11}-\Pi_{12}-\Pi_{44}\right)\left(L_{3311} \sigma_{11}+L_{3322} \sigma_{22}\right)
\end{aligned}
$$

Finding a combination of crystallographic orientations of $L_{3311}$ and $L_{3322}$ that is scalar-valued corresponds to finding the conditions under which the piezoresistor is insensitive to the in-plane biaxial stress orientation.

From the viewpoint of tensor calculus, these considerations provide a state-of-the-art physical basis for and insight into the classical PT theory.

\subsection{Piezoresistor responding only to transverse normal stress}

In Sects. 4.3 and 4.4, we apply the concept described in Sect. 4.1. We consider a piezoresistor subject to biaxial normal and shear stresses as shown in Fig. 8. The relative resistance change for the current density and electrical field in the $x_{3}$ direction is given by Eq. (10), which neglects the dimensional change due to the elastic deformation. If we consider the plane stress in the $\left(x_{1}, x_{2}\right)$ plane, Eqs. (10) and (23) lead to

$$
\begin{aligned}
\frac{\Delta \rho_{33}}{\rho_{0}}= & \left(\Pi_{11}+2 \Pi_{12}-\Pi_{44}\right) \sigma \\
& +\left(\Pi_{11}-\Pi_{12}-\Pi_{44}\right)\left[L_{3311}\left(\sigma_{11}-\sigma\right)+L_{3322}\left(\sigma_{22}-\sigma\right)-L_{3333} \sigma+2 L_{3312} \sigma_{12}\right] .
\end{aligned}
$$

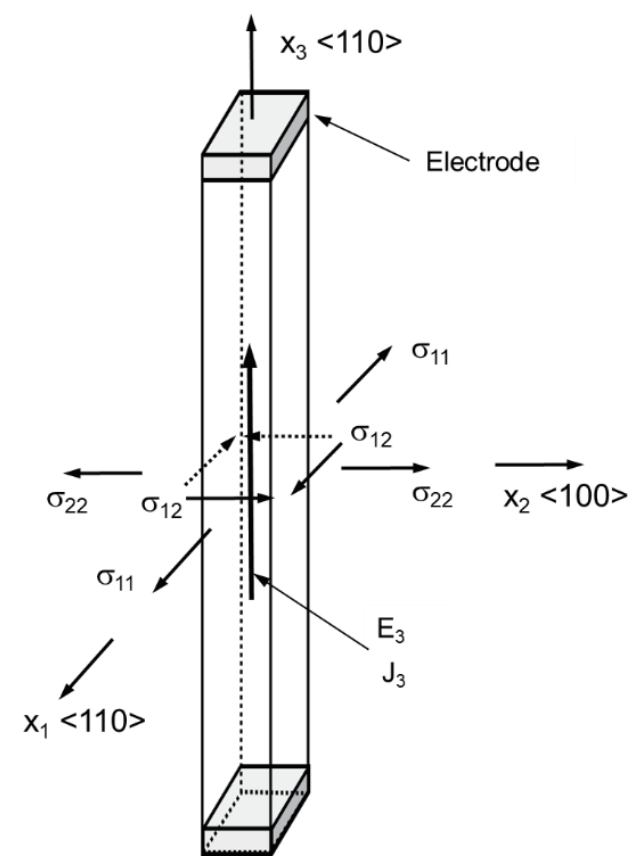

Fig. 8. Piezoresistor subjected to biaxial normal and shear stresses [relative resistance change for the current density and electrical field in the $x_{3}$ direction with the plane stress in the $\left(x_{1}, x_{2}\right)$ plane]. 
The response depends on the three plane stress components $\sigma_{11}, \sigma_{22}$, and $\sigma_{12}$ in the $\left(x_{1}, x_{2}\right)$ plane. As a particular case, the piezoresistor responds only to the biaxial normal stresses $\sigma_{11}$ and $\sigma_{22}$ in the $x_{1}$ and $x_{2}$ directions, respectively, if the $x_{1}$ and $x_{3}$ directions are $<110>$ and the $x_{2}$ direction is $<100>$. In this case, the three Euler angles $(\sigma, \theta, \phi)$ are $\left(\psi=0, \theta=\frac{\pi}{4}, \phi=0\right)$. Then, Eq. (3) becomes Eq. (29).

$$
\begin{array}{cccc} 
& x_{1} & x_{2} & x_{3} \\
X_{1} & \frac{1}{\sqrt{2}} & 0 & -\frac{1}{\sqrt{2}} \\
X_{2} & 0 & 1 & 0 \\
X_{3} & \frac{1}{\sqrt{2}} & 0 & \frac{1}{\sqrt{2}}
\end{array}
$$

The fourth-rank tensor components $L_{i j k l}$ obtained from Eq. (10) are $L_{3311}=L_{3333}=\frac{1}{2}$ and $L_{3311}=$ $L_{3312}=0$. Indeed, the piezoresistor does not respond to the in-plane shear stress component $\sigma_{12}$ because $L_{3312}=0$. Thus, Eq. (26) leads to

$$
\frac{\Delta \rho_{33}}{\rho_{0}}=\frac{1}{2}\left(\Pi_{11}+\Pi_{12}-\Pi_{44}\right) \sigma_{11}+\Pi_{12} \sigma_{22}
$$

It is evident that Eq. (30) coincides with Eq. (11) of Pfann and Thurston. ${ }^{(8)}$

Furthermore, the orthogonal orientations insensitive to both $\sigma_{22}$ and $\sigma_{12}$ can be found by choosing the Euler angles as $\left(\psi=\frac{\pi}{2}, \theta=\frac{\pi}{2}+\alpha, \phi=\frac{\pi}{4}\right)$. Then, Eq. (3) becomes Eq. (31).

$$
\begin{array}{cccc} 
& x_{1} & x_{2} & x_{3} \\
X_{1} & -\frac{1}{\sqrt{2}} & -\frac{\sin \alpha}{\sqrt{2}} & -\frac{\cos \alpha}{\sqrt{2}} \\
X_{2} & -\frac{1}{\sqrt{2}} & \frac{\sin \alpha}{\sqrt{2}} & \frac{\cos \alpha}{\sqrt{2}} \\
X_{3} & 0 & \cos \alpha & -\sin \alpha
\end{array}
$$

The fourth-rank tensor components $L_{i j k l}$ obtained from Eq. (31) are $L_{3311}=\frac{1}{2} \cos ^{2} \alpha$, $L_{3322}=\frac{3}{2} \cos ^{2} \alpha \sin ^{2} \alpha, L_{3312}=0$, and $L_{3333}=\frac{1}{2}\left(\cos ^{4} \alpha+\sin ^{4} \alpha\right)$. Therefore, Eq. (28) leads to 


$$
\frac{\Delta \rho_{33}}{\rho_{0}}=\Pi_{12}\left(1-\frac{\cos ^{2} \alpha}{2 C}\right) \sigma_{11}+\Pi_{12}\left(1-\frac{3 \sin ^{2} 2 \alpha}{8 C}\right) \sigma_{22} .
$$

where $C=-\frac{\Pi_{12}}{\Pi_{11}+\Pi_{12}-\Pi_{44}}$.

Equation (32) is identically insensitive to $\sigma_{12}$. The combination of Eqs. (23) and (24) leads to a physical basis for the problem that a piezoresistor only responds to $\sigma_{11}$ because $L_{3322}$ satisfies Eq. (24), i.e., $L_{3322}=\frac{3}{2} \cos ^{2} \alpha \sin ^{2} \alpha=C$. Indeed, if we choose $\sin ^{2} \alpha=\frac{8 C}{3}$, then Eq. (32) is insensitive to $\sigma_{22}$. It is evident that Eq. (32) coincides with Eq. (13) of Pfann and Thurston. ${ }^{(8)}$

\subsection{Piezoresistor having zero transverse normal stress sensitivity}

The relative resistance change for the current density and electrical field in the $x_{3}$ direction is given by Eqs. (10) and (23), which neglect the dimensional change due to elastic deformation. If we consider the plane stress in the $\left(x_{1}, x_{3}\right)$ plane shown in Fig. 9, Eqs. (10) and (23) lead to

$$
\begin{aligned}
\frac{\Delta \rho_{33}}{\rho_{0}}= & \left(\Pi_{11}+2 \Pi_{12}-\Pi_{44}\right) \sigma+\Pi_{44} \sigma_{33} \\
& +\left(\Pi_{11}-\Pi_{12}-\Pi_{44}\right)\left[L_{3311}\left(\sigma_{11}-\sigma\right)+L_{3322} \sigma-L_{3333}\left(\sigma_{33}-\sigma\right)+2 L_{3313} \sigma_{13}\right] .
\end{aligned}
$$

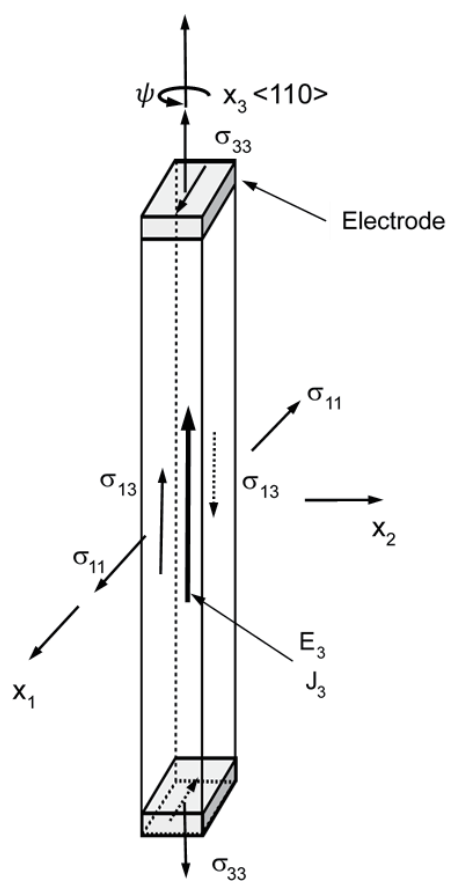

Fig. 9. Piezoresistor subjected to biaxial normal and shear stresses [relative resistance change for the current density and electrical field in the $x_{3}$ direction with the plane stress in the $\left(x_{1}, x_{3}\right)$ plane]. 
The orthogonal orientations insensitive to both $\sigma_{33}$ and $\sigma_{13}$ can be found by choosing the Euler angles as $\left(\psi, \theta=\frac{\pi}{4}, \phi=0\right)$. Then, Eq. (3) becomes Eq. (34).

$$
\begin{array}{cccc} 
& x_{1} & x_{2} & x_{3} \\
X_{1} & \frac{\cos \psi}{\sqrt{2}} & \sin \psi & -\frac{\cos \psi}{\sqrt{2}} \\
X_{2} & -\frac{\sin \psi}{\sqrt{2}} & \cos \psi & \frac{\sin \psi}{\sqrt{2}} \\
X_{3} & \frac{1}{\sqrt{2}} & 0 & \frac{1}{\sqrt{2}}
\end{array}
$$

The angle $\psi$ is an arbitrary rotation around the $\mathrm{x}_{3}$ axis perpendicular to the $\{110\}$ plane. The fourth-rank tensor components $L_{i j k l}$ obtained from Eq. (34) are $L_{3313}=0, L_{3311}=\frac{1}{2} \cos ^{2} \psi$, $L_{3322}=\frac{1}{2} \sin ^{2} \psi$, and $L_{3333}=\frac{1}{2}$. Indeed, the piezoresistor does not respond to the in-plane shear stress component $\sigma_{13}$ because $L_{3313}=0$. Equation (33) is reduced to

$$
\frac{\Delta \rho_{33}}{\rho_{0}}=\Pi_{12}\left(1-\frac{1}{6 C}\right) \sigma_{33}+\Pi_{12}\left(1-\frac{\cos ^{2} \psi}{2 C}\right) \sigma_{11},
$$

where $C=-\frac{\Pi_{12}}{\Pi_{11}-\Pi_{12}-\Pi_{44}}$.

Equation (35) is identically insensitive to $\sigma_{13}$. The combination of Eqs. (23) and (24) leads to a physical basis for the problem that a piezoresistor only responds to $\sigma_{33}$ because $L_{3311}$ satisfies Eq. (24), i.e., $L_{3311}=\frac{1}{2} \cos ^{2} \psi=C$. Indeed, if we choose $\cos ^{2} \psi=2 C$, then Eq. (35) is insensitive to $\sigma_{11}$. Thus, the piezoresistor responds only to the longitudinal normal stress $\sigma_{33}$ and has zero transverse normal stress sensitivity. It is evident that Eq. (35) coincides with Eq. (20) of Pfann and Thurston. ${ }^{(8)}$

\subsection{Piezoresistor insensitive to stress orientation}

In this section, we apply the concept described in Sect. 4.2. Figure 10 shows a piezoresistor in the form of an ell with two in-plane perpendicular arms, where $x_{1}$ and $x_{2}$ denote the directions of the arms. If we consider the plane stresses $\sigma_{11}, \sigma_{22}$, and $\sigma_{12}$ in the $\left(x_{1}, x_{2}\right)$ plane, then Eq. (10) leads to 


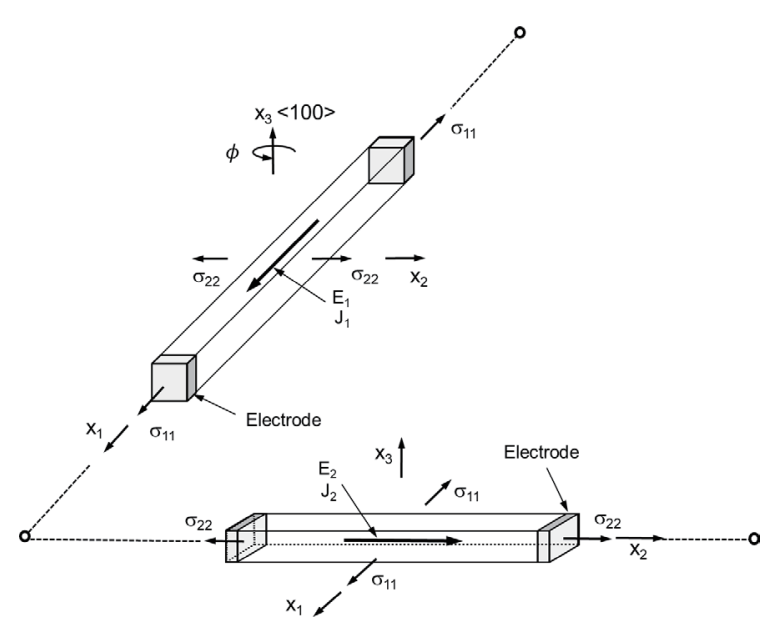

(a)

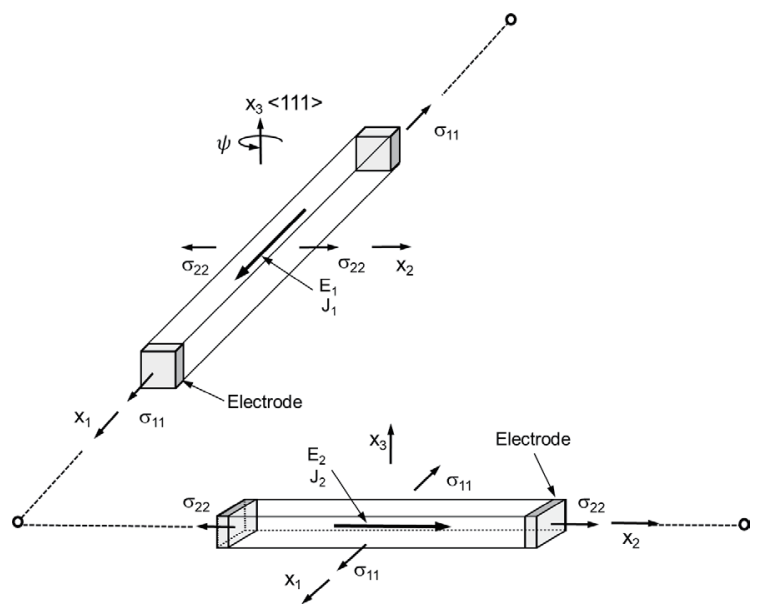

(b)

Fig. 10. Piezoresistor made in the form of an ell with the in-plane two perpendicular arms: (a) $x_{3} / /\{100\}$, (b) $x_{3} / /\{111\}$, $x_{1}$ and $x_{2}$ denote the directions of the arms.

$$
\begin{aligned}
\frac{\Delta \rho_{11}}{\rho_{0}}+\frac{\Delta \rho_{22}}{\rho_{0}}= & \frac{2}{3}\left(\Pi_{11}+2 \Pi_{12}+\Pi_{44}\right)\left(\sigma_{11}+\sigma_{22}\right) \\
& -\frac{\left(\Pi_{11}-\Pi_{12}-\Pi_{44}\right)}{3}\left(L_{1133}+L_{2233}\right)\left(\sigma_{11}+\sigma_{22}\right) \\
& +\frac{\left(\Pi_{11}-\Pi_{12}-\Pi_{44}\right)}{3}\left[\left(L_{1111}+L_{1122}\right)\left(2 \sigma_{11}-\sigma_{22}\right)\right. \\
& \left.+\left(L_{1122}+L_{2222}\right)\left(2 \sigma_{22}-\sigma_{11}\right)\right] .
\end{aligned}
$$

A piezoresistor insensitive to the orientations of $\sigma_{11}$ and $\sigma_{22}$ can be obtained from Eq. (10) by choosing the Euler angles as ( $\psi=0, \theta=0, \phi)$. Then, Eq. (3) becomes Eq. (37).

$$
\begin{array}{cccc} 
& x_{1} & x_{2} & x_{3} \\
X_{1} & \cos \phi & \sin \phi & 0 \\
X_{2} & -\sin \phi & \cos \phi & 0 \\
X_{3} & 0 & 0 & 1
\end{array}
$$

The angle $\phi$ is an arbitrary rotation around the $x_{3}$ axis perpendicular to the $\{100\}$ plane. The fourth-rank tensor components $L_{i j k l}$ obtained from Eq. (37) are $L_{1111}=L_{2222}=\cos ^{4} \phi+\sin ^{4} \phi$, $L_{1122}=2 \cos ^{2} \phi \sin ^{2} \phi$, and $L_{1133}=L_{2233}=0$. Equation (10) is reduced to

$$
\frac{\Delta \rho_{11}}{\rho_{0}}+\frac{\Delta \rho_{22}}{\rho_{0}}=\left(\Pi_{11}+\Pi_{12}\right)\left(\sigma_{11}+\sigma_{22}\right) .
$$


Equation (38) is identically insensitive to the orientations of $\sigma_{11}$ and $\sigma_{22}$. It is evident that Eq. (38) coincides with Eq. (23) of Pfann and Thurston. ${ }^{(8)}$ An alternative solution of Eq. (38) is straightforward and is obtained from Eq. (27) as

$$
\frac{\Delta \rho_{m m}}{\rho_{0}}-\frac{\Delta \rho_{33}}{\rho_{0}}=\left(\Pi_{11}+\Pi_{12}\right)\left(\sigma_{11}+\sigma_{22}\right) .
$$

An alternative piezoresistor insensitive to the orientations of $\sigma_{11}$ and $\sigma_{22}$ can be obtained from Eq. (27) by choosing the Euler angles as $\left(\psi, \theta=\cos ^{-1} \frac{1}{\sqrt{3}}, \phi=\cos ^{-1} \frac{1}{\sqrt{2}}\right)$. Then, Eq. (3) becomes Eq. (40).

$$
\begin{array}{cccc} 
& x_{1} & x_{2} & x_{3} \\
X_{1} & \frac{\cos \psi}{\sqrt{6}}-\frac{\sin \psi}{\sqrt{2}} & \frac{\cos \psi}{\sqrt{6}}+\frac{\sin \psi}{\sqrt{2}} & -\sqrt{\frac{2}{3}} \cos \psi \\
X_{2} & -\frac{\sin \psi}{\sqrt{6}}-\frac{\cos \psi}{\sqrt{2}} & -\frac{\sin \psi}{\sqrt{6}}+\frac{\cos \psi}{\sqrt{2}} & \sqrt{\frac{2}{3}} \sin \psi \\
X_{3} & \frac{1}{\sqrt{3}} & \frac{1}{\sqrt{3}} & \frac{1}{\sqrt{3}}
\end{array}
$$

The angle $\psi$ is an arbitrary rotation around the $x_{3}$ axis perpendicular to the $\{111\}$ plane as shown in Fig. 10(b). The fourth-rank tensor components $L_{i j k l}$ obtained from Eq. (40) are $L_{1111}=L_{1122}=\frac{1}{2}, L_{2222}=\frac{1}{6}$, and $L_{1133}=L_{2233}=L_{3333}=\frac{2}{3}$. Equation (10) leads to

$$
\frac{\Delta \rho_{11}}{\rho_{0}}+\frac{\Delta \rho_{22}}{\rho_{0}}=\frac{1}{3}\left(2 \Pi_{11}+4 \Pi_{12}+\Pi_{44}\right)\left(\sigma_{11}+\sigma_{22}\right) .
$$

Equation (41) is identically insensitive to the orientations of $\sigma_{11}$ and $\sigma_{22}$. It is evident that Eq. (41) coincides with Eq. (24) of Pfann and Thurston. ${ }^{(8)}$ Similarly to Eq. (39), an alternative solution of Eq. (41) can be easily obtained from Eq. (27).

\section{Conclusions}

We have derived a single piezoresistance tensor equation that clearly indicates the physical role of the state of multiaxial stress in the cubic crystal anisotropy of piezoresistance. The piezoresistance tensor equation for cubic crystal symmetry can be expressed in terms of the three independent components of the principal piezoresistance tensor, the isotropic and 
deviatoric stress tensors, and the fourth-rank coordinate transformation tensor. To demonstrate the validity of the proposed tensor equation, an experiment on the single-crystal silicon piezoresistance stress gauge subjected to multiaxial stress was carried out. Furthermore, the proposed piezoresistance tensor equation can give a physical basis for the typical single-crystal silicon piezoresistive stress transducers derived by Pfann and Thurston.

\section{Acknowledgments}

This work was supported by JSPS KAKENHI Grant Number JP16H03819.

\section{References}

1 T. Toriyama and S. Sugiyama: Appl. Phys. Lett. 81 (2002) 2797.

2 T. Toriyama and S. Sugiyama: J. Microelectromech. Syst. 11 (2002) 598.

3 A. A. Barlian, W. T. Park, J. R. Mallon, Jr., A. J. Rastegar, and B. L. Pruitt: Proc. IEEE 97 (2009) 513.

4 H. P. Phan, D. V. Dao, P. Tanner, L. Wang, N. T. Nguyen, Y. Zhu, and S. Dimitrijev: Appl. Phys. Lett. 104 (2014) 111905.

5 H. P. Phan, D. V. Dao, K. Nakamura, S. Dimitrijev, and N. T. Nguyen: J. Microelectromech. Syst. 24 (2015) 6.

6 C. S. Smith: Phys. Rev. 94 (1954) 42.

7 C. S. Smith: Solid State Phys. 6 (1958) 232.

8 W. G. Pfann and R. N. Thurston: J. Appl. Phys. 32 (1961) 2008.

9 O. N. Tufte and E. L. Stelzer, J. Appl. Phys. 34 (1963) 313.

10 O. N. Tufte and E. L. Stelzer: Phys. Rev. 133 (1964) A1705.

11 N. Matsuzuka and T. Toriyama: J. Appl. Phys. 108 (2010) 064902.

12 H. Margenau and G. M. Murphy: The Mathematics of Physics and Chemistry (Young Press, 2009) 2nd. ed., p. 289.

13 S. Nemat-Nasser: Plasticity (Cambridge University Press, 2004) p. 16.

14 J. F. Nye: Physical Properties of Crystals (Oxford University Press, Oxford, 1990) p. 134.

15 J. J. Wortman and R. A. Evans: J. Appl. Phys. 36 (1965) 153.

16 J. Richter, J. Pedersen, M. Brandbyge, E. V. Thomsen, and O. Hansen: J. Appl. Phys. 104 (2008) 023715.

17 H. Jeffreys and B. Jeffreys: Methods of Mathematical Physics (Cambridge University Press, 1972) 3rd ed., p. 88.

\section{Appendix}

\section{A. Isotropic and deviatoric fourth-rank tensors ${ }^{(17)}$}

Components of the isotropic tensor are invariant with respect to the coordinate transformation. We can make an isotropic fourth-rank tensor from an arbitrary fourth-rank tensor with the symmetry of $T_{i j k l}=T_{j i k l}=T_{i j k}$,

$$
\left(T_{i j k l}\right)_{\text {isotropic }}=T_{1} \delta_{i j} \delta_{k l}+2 T_{2} I_{i j k l},
$$

where

$$
T_{1}=\frac{1}{15}\left(2 T_{p p q q}-T_{p q p q}\right),
$$




$$
T_{2}=\frac{1}{30}\left(3 T_{p q p q}-T_{p p q q}\right)
$$

A deviatoric part of the tensor can be obtained by subtracting the isotropic part from the original tensor.

$$
\left(T_{i j k l}\right)_{\text {deviatoric }}=T_{i j k l}-\left(T_{i j k l}\right)_{\text {isotropic }}
$$

Substituting Eq. (4), i.e., $\pi_{i j k l}=\hat{\Pi}_{1} \delta_{i j} \delta_{k l}+2 \hat{\Pi}_{2} I_{i j k l}+\hat{\Pi}_{3} L_{i j k l}$ into Eqs. (A2) and (A3), we obtain

$$
\begin{gathered}
\pi_{p p q q}=\hat{\Pi}_{1} \delta_{p p} \delta_{q q}+2 \hat{\Pi}_{2} I_{p p q q}+\hat{\Pi}_{3} L_{p p q q}=9 \hat{\Pi}_{1}+6 \hat{\Pi}_{2}+3 \hat{\Pi}_{3}, \\
\pi_{p q p q}=\hat{\Pi}_{1} \delta_{p q} \delta_{p q}+2 \hat{\Pi}_{2} I_{p q p q}+\hat{\Pi}_{3} L_{p q p q}=3 \hat{\Pi}_{1}+12 \hat{\Pi}_{2}+3 \hat{\Pi}_{3}, \\
T_{1}=\hat{\Pi}_{1}+\frac{1}{5} \hat{\Pi}_{3} \text { and } T_{2}=\hat{\Pi}_{2}+\frac{1}{5} \hat{\Pi}_{3} .
\end{gathered}
$$

Note that $L_{p p q q}=L_{p q p q} \equiv \sum_{L=1}^{3}\left(a_{L p}\right)^{2}\left(a_{L q}\right)^{2}=3$.

Thus, Eqs. (A1) and (A4) can be rewritten by using Eqs. (A2), (A3), (A5), (A6), and (A7),

$$
\begin{gathered}
\left(\pi_{i j k l}\right)_{\text {isotropic }}=\left(\hat{\Pi}_{1}+\frac{1}{5} \hat{\Pi}_{3}\right) \delta_{i j} \delta_{k l}+2\left(\Pi_{2}+\frac{1}{5} \Pi_{3}\right) I_{i j k l}, \\
\left(\pi_{i j k l}\right)_{\text {deviatoric }}=\hat{\Pi}_{3}\left[L_{i j k l}-\frac{1}{5}\left(\delta_{i j} \delta_{k l}+2 I_{i j k l}\right)\right] .
\end{gathered}
$$

\section{B. Derivations of Eqs. (24) and (25)}

Here, the tensor summation convention does not apply to the Greek indices. The piezoresistance in the cubic single crystal subjected to multiaxial stress with the electrical field and current density vectors both parallel to the $x_{\alpha}$ direction is given as

$$
\begin{aligned}
\frac{\Delta \rho_{\alpha \alpha}}{\rho_{0}}= & \frac{1}{3}\left(\Pi_{11}+2 \Pi_{12}\right) \sigma_{k k}+\Pi_{44}\left(\sigma_{\alpha \alpha}-\frac{1}{3} \sigma_{k k}\right) \\
& +\left(\Pi_{11}-\Pi_{12}-\Pi_{44}\right) L_{\alpha \alpha i j}\left(\sigma_{i j}-\frac{1}{3} \sigma_{k k} \delta_{i j}\right),
\end{aligned}
$$


where the deviatoric part of Eq. (23) is

$$
\begin{aligned}
& \Pi_{44}\left[\sigma_{\alpha \alpha}-\frac{1}{3}\left(\sigma_{11}+\sigma_{22}+\sigma_{33}\right)\right] \\
& +\left(\Pi_{11}-\Pi_{12}-\Pi_{44}\right)\left(L_{\alpha \alpha 11} \sigma_{11}+L_{\alpha \alpha 22} \sigma_{22}+L_{\alpha \alpha 33} \sigma_{33}\right. \\
& \left.+2 L_{\alpha \alpha 12} \sigma_{12}+2 L_{\alpha \alpha 23} \sigma_{23}+2 L_{\alpha \alpha 31} \sigma_{31}\right) \\
& \quad-\frac{1}{3}\left(\Pi_{11}-\Pi_{12}-\Pi_{44}\right)\left(\sigma_{11}+\sigma_{22}+\sigma_{33}\right)
\end{aligned}
$$

and the terms related to the normal stress component $\sigma_{\beta \beta}$ are

$$
\begin{aligned}
& -\frac{1}{3} \Pi_{44} \sigma_{\beta \beta}+\left(\Pi_{11}-\Pi_{12}-\Pi_{44}\right)\left(L_{\alpha \alpha \beta \beta}-\frac{1}{3}\right) \sigma_{\beta \beta} \text { for } \alpha \neq \beta, \\
& \frac{2}{3} \Pi_{44} \sigma_{\beta \beta}+\left(\Pi_{11}-\Pi_{12}-\Pi_{44}\right)\left(L_{\alpha \alpha \beta \beta}-\frac{1}{3}\right) \sigma_{\beta \beta} \text { for } \alpha=\beta .
\end{aligned}
$$

The hydrostatic part of Eq. (23) is scalar-invariant and always responds to hydrostatic stress regardless of the crystallographic orientation.

$$
\frac{1}{3}\left(\Pi_{11}+2 \Pi_{12}\right)\left(\sigma_{11}+\sigma_{22}+\sigma_{33}\right)
$$

Therefore, making a piezoresistor unresponsive to the normal stress component $\sigma_{\beta \beta}$ corresponds to cancelling $\sigma_{\beta \beta}$ appearing in Eq. (B4), which is derived from Eqs. (B2) and (B3):

$$
\begin{aligned}
& -\frac{1}{3} \Pi_{44} \sigma_{\beta \beta}+\left(\Pi_{11}-\Pi_{12}-\Pi_{44}\right)\left(L_{\alpha \alpha \beta \beta}-\frac{1}{3}\right) \sigma_{\beta \beta}=-\frac{1}{3}\left(\Pi_{11}+2 \Pi_{12}\right) \sigma_{\beta \beta} \text { for } \alpha \neq \beta, \\
& \frac{2}{3} \Pi_{44} \sigma_{\beta \beta}+\left(\Pi_{11}-\Pi_{12}-\Pi_{44}\right)\left(L_{\alpha \alpha \beta \beta}-\frac{1}{3}\right) \sigma_{\beta \beta}=-\frac{1}{3}\left(\Pi_{11}+2 \Pi_{12}\right) \sigma_{\beta \beta} \text { for } \alpha=\beta
\end{aligned}
$$

Thus, we obtain the following set of crystallographic orientations that satisfy Eqs. (B5) and (B6):

$$
\begin{aligned}
& L_{\alpha \alpha \beta \beta}=-\frac{\Pi_{12}}{\Pi_{11}-\Pi_{12}-\Pi_{44}} \text { for } \alpha \neq \beta, \\
& L_{\alpha \alpha \beta \beta}=-\frac{\Pi_{12}+\Pi_{44}}{\Pi_{11}-\Pi_{12}-\Pi_{44}} \text { for } \alpha=\beta .
\end{aligned}
$$




\section{About the Authors}

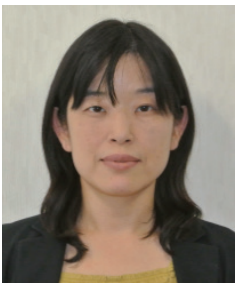

Taeko Ando received her B.E. degree in aerospace engineering and her M.S. and Ph.D. degrees in microsystem engineering from Nagoya University, Japan, in 1996, 1999, and 2001, respectively. From 2001 to 2008, she was an assistant professor and from 2008 to 2009, a senior lecturer at Nagoya University, Japan. Since 2009, she has been an associate professor at Ritsumeikan University. Her research interests are in the physical properties of MEMS material in a micro/nanoscale.

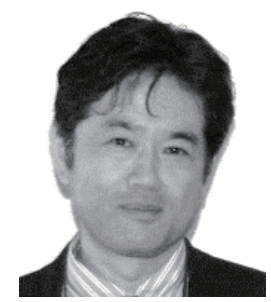

Toshiyuki Toriyama received his Ph.D. degree from Kyushu University, Japan, in 1994. Since 2004, he has been a professor at Ritsumeikan University, Japan. His research interests are in piezoresistance materials and microscale turbomachinery. 